\title{
Exploring Lost and Found in Future Images of Energy Transitions: Towards a Bridging Practice of Provoking and Affirming Design
}

\author{
HESSELGREN Mia ${ }^{a^{*}}$; ERIKSSON Elina ${ }^{\mathrm{a}}$; WANGEL Josefin ${ }^{\mathrm{b}}$ and BROMS Loove ${ }^{\mathrm{a}}$ \\ a KTH Royal Institute of Technology \\ ${ }^{b}$ SLU Swedish University of Agricultural Sciences \\ * Corresponding author e-mail: miahes@kth.se \\ doi: 10.21606/dma.2018.324
}

\begin{abstract}
We need to transition our society in a more sustainable direction, for example through enormous cuts in carbon emissions. Yet this future is hard to envision and work towards. In this project, with a transition design posture, we have designed tools that we believe can be useful to initiate dialogues and reflections for the future. In particular we are interested in using the bridging between provocative and affirmative design as a way to explore and articulate what people see as the lost and found of such a transition. In this paper, we present a study where we used a practice lens to address one possible low carbon future through a provocation workshop. We present our methodology, the tentative tools we used during the workshop and the experiences as expressed by the workshop participants.
\end{abstract}

sustainability transitions; transition design posture; provocative and affirmative design; lost and found

\section{Introduction}

Every day, we are bombarded with news of extreme weather events, species extinction and land devastation. A search in the Swedish media archive shows that between 2014 and 2017 there was an average of 21000 articles per year in Swedish newspapers on climate change related topics ${ }^{1}$. We know that several of the planetary boundaries have been overstepped (Steffen et al., 2015) and the scientific community is univocal in its agreement that climate change is real and with human origin (Stocker et al., 2013). We also know that a failure to keep global warming under 2 degrees Celsius,

\footnotetext{
${ }^{1}$ The articles were identified through searching for words including "klimat" (climate) in Swedish printed media published 2014-2017. Press releases and news agencies were excluded. The search was done 2017-11-05.
}

This work is licensed under a Creative Commons Attribution-NonCommercial-Share Alike 4.0 
or, preferably, 1.5 degrees, most probably will lead to changes in climate systems with "severe, pervasive and irreversible impacts for people and ecosystems" (IPCC 2014, p.8). Yet, the transition to a more sustainable society with less carbon emissions is moving slowly. The UN emissions report of 2017 shows that the gap between the reductions needed and the national pledges made in Paris is "alarmingly high" (UNEP, 2017).

This is not a new concern. Back in 2001, Norgaard (2011) did a one year ethnographic study in a Norwegian village and showed that despite the diminishing weeks of snow cover that affected the villagers' livelihood, based on ski tourism, her respondents still avoided talking or thinking about climate change. Norgaard's study showed that it is not lack of information that hindered action from the Norwegian villagers, but that people tend to shut out information that makes them uncomfortable. Through avoiding negative emotions and refraining from thinking about the future, climate change is actively (although not consciously) made into a "back-of-the-mind" issue. However, for a transition to happen, climate change needs to be transformed to a "front-of-themind" issue in politics (Giddens, 2011) as well as in everyday life. But how do we overcome the mechanisms of denial?

Studies on climate change communication have shown that too much alarmism depersonalizes the problem and makes it harder for individuals to engage and act (Ereaut \& Segnit, 2006; Lowe, 2006). Moreover, while alarmist accounts could indeed induce emotions like fear, which could be a driver for pro-environmental behaviour, many people suffer from a perceived lack of agency and alternatives. Fear, rather than motivating people to act, thus lead to feelings of helplessness, hopelessness and inaction (Kollmuss \& Agyeman, 2002).

Weber (2010) suggests that for individuals to become more engaged in climate change concretisation is needed, both by making climate change consequences more specific, as well as moving these closer in time and place. Schneider-Mayerson (2017) instead points to the lack of positive images of what low carbon futures might look like, making action hard to encourage. Furthermore, Randall (2009) describes the parallel narratives on climate change, where the problems lead to catastrophic losses, but where the solution narratives are often devoid of loss. Ignoring loss when working with climate change risk effects can hold back change efforts, or distract us from action, and result instead in rejection and avoidance, manic activity, idealization of lost things, or focusing on false solutions (ibid.).

In this paper, we seek to explore how transition design and futures studies could be used as empowering tools in relation to climate change mitigation. In particular we explore ways to identify and articulate what people see as lost and found in the transition to a low-carbon society, to investigate if there are ways to confront the lost, so this seems less threatening, and to mentally and emotionally invest in the found, to make the transition more appealing.

\section{Framing our project as transition design}

Sustainability transitions are extraordinarily complex, future oriented, abstract and global, as compared to less challenging problems that tend to be more immediate, visible and local (Geels, 2010). This implies that sustainability transitions need to be addressed by transdisciplinary approaches in which not only different scientific disciplines are involved but also other types of knowledge cultures such as practice based, tacit and lay knowledge (Miller et al., 2008; Robinson, 2004; 2008). Also Stirling (2011) urges for transdisciplinary approaches to open up for plural possibilities of transformations.

Transition design is an emerging design research field that aims at engaging design practice in exploring and enabling transitions towards more sustainable futures. It acknowledges that design can act as catalyst for change, even for complex systems, and suggests a role for designers as change agents (Irwin, Kossoff, \& Tonkinwise, 2015). Irwin, Kossoff and Tonkinwise (2015) present four "building blocks" of transition design: 1) visions for transitions, 2) theories of change, 3) posture and 
mindset, and 4) new ways of designing. We elaborate on these building blocks in this paper, although in a different order. First, we explore what an interlinking of posture and mindset with theories of change brings us (focusing on socio-technical transition theories and social practice theories, respectively). Thereafter, we continue with exploring ways of designing and developing visions of transition.

\subsection{Mindset and posture from within theories of change}

Transition design advocates a mindset and posture that is precautionary, encouraging an explorative, reflexive and critical stance rather than aiming for optimized solutions (Tonkinwise, 2016). Transition design also advocates a mindset that is participatory, promoting collaborative efforts which acknowledge lay and tacit knowledge and know-how alongside professional and academic knowledge. A precautionary mindset and posture resonates well with socio-technical transition theories, such as transition management, as these promote reflexive learning for adaptive governance through visioning and experimentation (Kemp, Loorbach, \& Rotmans, 2007). Transition management is also in line with a participatory mindset and posture but has traditionally focused on engaging people-as-professionals rather than as lay experts or experts on everyday life.

To understand the local, place-based and situated, and to engage people-as-people, a practiceoriented approach is useful. Practices are routinized activities carried out in everyday life (Reckwitz, 2002). Social practice theories focus on how people's everyday practices are shaped in the social context. Using social practice theories as a way to understand how to change "behaviours", is to acknowledge that the possibility of change lies in the emergence and maintenance of the practice itself (Warde, 2005). Social practice theories constitute one field of the transition theories and as such, is the one closest to people and their behaviour (Geels, 2010; Shove \& Walker, 2010). They are particularly well-suited as a basis for transition design since the starting point for design has traditionally been in understanding (and influencing) the needs and wants of people (Forty, 1986). Also, the flatter ontology of social practice theories suits the design researcher, trained in messiness (Schön, 1983).

When aiming at supporting sustainability transitions, attention can be paid to vertical relations, as in the multi-level perspective (Geels, 2010), or to horizontal trajectories and interconnections of practices (Shove \& Walker, 2010). Transition design could be used to mediate between sociotechnical transition theories with their top-down hierarchical approaches and, and social practice theories with their bottom-up focus on everyday life and flat ontology. The capacity to iterate between concrete details of everyday life and more abstract concepts is a characteristic of most design practices (Nelson \& Stolterman, 2003). However, what is specific in transition design is the connection to more macro-scaled societal structures and processes. Hence we believe that transition designers as change agents can thus operate at multiple levels of scale, including time and place.

\subsection{Ways of designing and visions: Provoking and affirming design}

To even further connect with people and their everyday practices, we believe co-design can be productive. Co-design, as a design research approach, is based in a participatory mindset where users are viewed as partners (Sanders, 2008). This partnership can be formed with different outsets (for or with the user) and different timescales in mind (near or far futures), forming a number of different design research approaches (Sanders \& Stappers, 2014). In co-design, as ways to empower creativity amongst participants, bridging between pasts, presents and futures is often used, i.e. to discuss the present situation whilst referring to past experiences and then to envision future possibilities (Sanders \& Stappers, 2012). Co-design can also be implemented, together with users, to explore the connection between the tangible, present and local (such as dinner practices) with the more abstract, future and global (such as climate change impacts). This is difficult, however, and we argue that there is a need for tools to further help participants in these different movements - in time, in place and in possibilities. 
In the following we will use the concepts - provoking and affirming, respectively - to denote two design approaches we think are essential for transition design and which can be used in co-design workshops. We define provoking (or provocative) design approaches as those aiming at destabilizing and de-familiarizing (Bell, Blythe, \& Sengers, 2005) the taken for granted, routinized and "back-ofthe-mind" issues, in this way opening up for re-presenting and re-narrating processes. We define affirming (affirmative) design approaches as those aimed at supporting an exploration of the self, within ideas of present norms and practices, providing full preferential right of interpretation to the user. We acknowledge that there is tension between these concepts, but also see potential in using them for bridging purposes.

In this paper, we explore how such a bridging of provocative and affirmative design approaches could look in terms of concrete design tools, and how this can be used to explore more sustainable energy futures. We see design tools as research devices, which are links between objects and methods that can act as hinges between concepts and practices (Lury \& Wakeford, 2012). To use them in practice-based design research is to design and use artefacts to initiate thinking processes. More specifically we explore how such bridging research devices can be helpful to empower users to explore and articulate their images of more sustainable energy futures, in particular as a way to explore personal and societal lost and found in relation to sustainability transitions. This also includes the bridging of the tangible-present-local to the abstract-future-global, as discussed above.

\section{Using traces of practices}

\subsection{Introducing Empowering Energy Futures}

The material presented in this paper was developed in the research project Empowering Energy Futures carried out in Stockholm, Sweden 2015-2017. The team was interdisciplinary, including researchers and practitioners with backgrounds in industrial design, interaction design, humancomputer interaction, futures studies, systems analysis and graphic design. The overarching aim of the project was to explore people's images of the future from an energy transition perspective. In this paper, we focus on a subset of the activities in the project, where we sought to develop supportive tools for people to explore their own low carbon futures. Central to this effort was a 'provocation workshop' to which environmentally engaged participants were invited to explore the lost and found in such futures. Furthermore, the project developed an energy fiction, Vitiden, in the form of a manifesto and future archaeology with inspiration from design fiction. The energy fiction and its development will be presented in a separate forthcoming paper.

\subsection{What future and which everyday life?}

As a basis for the project we decided to use a scenario study by the Swedish Energy Agency called "Four Futures" [Fyra framtider] (Energimyndigheten, 2016). It explores and describes four possible futures in the years 2035 and 2050 with a focus on how the Swedish energy system could be developed. Each of the four scenarios - Forte, Vivace, Espressivo and Legato - is premised on a specific combination of driving forces: in Forte, economic growth and a strong export industry are the main priorities of Swedish society; Vivace builds on ecological modernization and export of Swedish green-tech; in Espressivo individual consumers and flexibility stand in focus; and for Legato ecological sustainability and global solidarity are the main concerns. These drivers influence not only the development of the energy system per se but also how industry, built environment, transport systems, and, to some extent, everyday life are organised. Each scenario is described in both qualitative and quantitative terms.

Since the aim of this project was to explore sustainable futures, and not just any futures, we decided to work solely with the scenario Legato, the only scenario in line with meeting the Paris agreement to keep global warming under 1.5 degrees. An initial analysis of Legato made it clear that while behavioural changes were mentioned, their descriptions were quite detached from everyday life, essentially making it difficult for people who were not energy systems experts to engage in this 
future and understand how it would affect them. Trawling for traces of practices in the text however resulted in a net list of eleven practices (or 'lifestyle changes'), including, for example, to bicycle more, to use car sharing, rental car or taxi instead of owning a car, and to work less or to work more locally. Looking closer at the list, it became clear that several of the practices focused on 'production' activities such as how and where to work, and that the rest mainly dealt with transport. Practices concerned with how we eat and reside were missing altogether, something that later found its explanation in the fact that Legato's impacts were mitigated through efficiency measures in production and infrastructure. Yet, reading between the lines, we could see that eating and residing as practices would also be affected by this particular future, if only indirectly, so we decided to add these to the list of practices. Another reason for this 'corruption' of data was that previous experience has shown that it is very difficult to engage people in discussions about everyday life while excluding large parts of it. Legato and its background data were also analysed to concretize what the changes in the energy system would imply in quantitative terms. This analysis showed that in Legato the carbon emission per person per year in 2050 would be 0.6 tonne of $\mathrm{CO}_{2} \mathrm{e}$, as compared to today's 10.8 , i.e. emission cuts by 94 per cent $^{2}$.

To understand what the low carbon practices in Legato could be like we decided to interview 'early adopters' and forerunners of sustainable lifestyles. From the net list of practices, we extracted four that we wanted to explore in more depth: "work less", "increase the level of self-sufficiency", "refrain from longer trips" and "refrain from environmentally burdening consumption". Indeed, such practices are entangled and hard to separate, and in the end, we identified and conducted contextual in-depth interviews with five respondents (Table 1).

Table 1 The five forerunners and their four entangled practices.

\begin{tabular}{|l|l|l|l|l|}
\hline & Work less & $\begin{array}{l}\text { Refrain from } \\
\text { consumption }\end{array}$ & $\begin{array}{l}\text { Refrain from } \\
\text { longer trips }\end{array}$ & $\begin{array}{l}\text { Increased self- } \\
\text { sufficiency }\end{array}$ \\
\hline Downshifter & $\mathrm{x}$ & $\mathrm{x}$ & $\mathrm{x}$ & \\
\hline Stopped flying & & & $\mathrm{x}$ & $\mathrm{x}$ \\
\hline Guerrilla-farmer/activist & & $\mathrm{x}$ & $\mathrm{x}$ & $\mathrm{x}$ \\
\hline Organic farmer & & $\mathrm{x}$ & & \\
\hline Simple living & $\mathrm{x}$ & $\mathrm{x}$ & & \\
\hline
\end{tabular}

Interviews were semi-structured and carried out by three of the researchers in the homes of the respondents. Interviews were audio recorded and notes and photographs were taken (see Figure 1 for examples). The insights into the forerunners' practices formed a basis for the subsequent design work. Their already existing practices could be considered potential practices for the many in the future and as such gave us insights into the tangible-present-local as possible departing points for the abstract-future-global.

\footnotetext{
${ }^{2}$ These calculations were made in several steps, including converting the partial-territorial system definition used in the construction of Legato to a consumption-based system definition more in line with the societal values of Legato. A comprehensive account on these calculations is available upon request.
} 

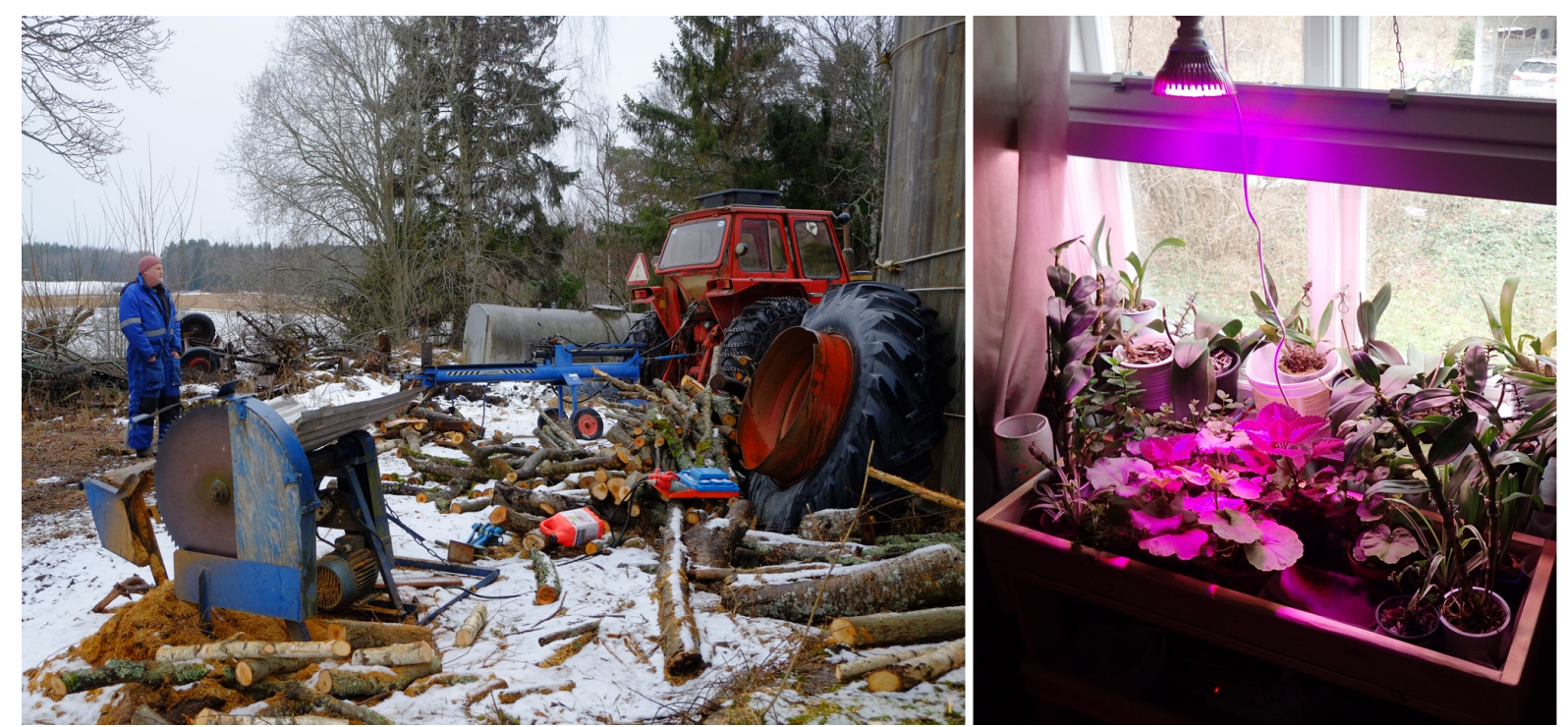

Figure 1 Examples from in-home-interviews with forerunners.

\section{Reconceptualizing the future through trigger materials}

When planning the workshop, we considered different kinds of materials that could be used to promote reflection and discussion amongst the participants. These were developed with emphasis on helping the participants to bridge the tangible-present-local to the abstract-future-global, and with particular emphasis on finding ways to balance the provocative with the affirmative. Another starting point was the wish to create a workshop process that meandered from the individual to the group and so on to more global issues. In the end, this resulted in the development of four different trigger materials used before and during the workshop.

\subsection{Trigger material 1}

The first trigger material was a homework assignment, where the invited participants were asked to make a climate footprint calculation before the workshop. For this purpose, the Swedish "Klimatkalkylatorn" 3 was chosen, which was suitable as it was readily available, fairly easy to use, included clear and simple result presentations with a coverage of the majority of the carbon emissions from everyday consumption, and was developed by trustworthy organizations. The idea with this trigger material was for it to function as a sensitizing material (Sanders \& Stappers, 2012), making the participants reflect beforehand on their current and past activities in relation to climate impact. Once in the workshop, the participants were asked to present their own results and were then presented with a brief presentation of the future we were to explore where the average carbon footprint per person and year would be 0.6 tons $\mathrm{CO}_{2} \mathrm{e}$.

\subsection{Trigger material 2}

The second trigger material was developed as a set of cards and a $2 \times 2$ matrix, with one dimension spanning from "happy" to "sad", and the other from "more in the future" to "less of in the future". The cards were inspired by photo elicitation (Harper, 2002). The specific pictures were chosen to represent possible configurations of the practices identified in Legato, the reference research and the interviews. Another selection criteria was to have an equal or close to equal representation of gender, class and ethnicity across pictures. We also sought to include ambiguous pictures, as well as more 'dystopian' pictures. The $2 \times 2$ matrix was developed inspired by explorative futures studies in which similar matrices are used to examine uncertainties.

\footnotetext{
${ }^{3}$ https://www.klimatkalkylatorn.se/ developed by SEl and WWF.
} 
The cards depicted different activities, practices and things - concrete enough to support associations, but still open for interpretation (See Figure 2). The idea was that the participants should use the cards to explore what their hopes and fears were in relation to a more sustainable future, essentially helping them explore and articulate lost and found. As a primer, the participants had the individual carbon footprints from the first trigger material as well as the targeted carbon footprint of $0.6 \mathrm{CO}_{2} \mathrm{e}$. After a period of self-reflection, the participants presented to the group some of their selected cards and placings, and described their feelings connected to these.

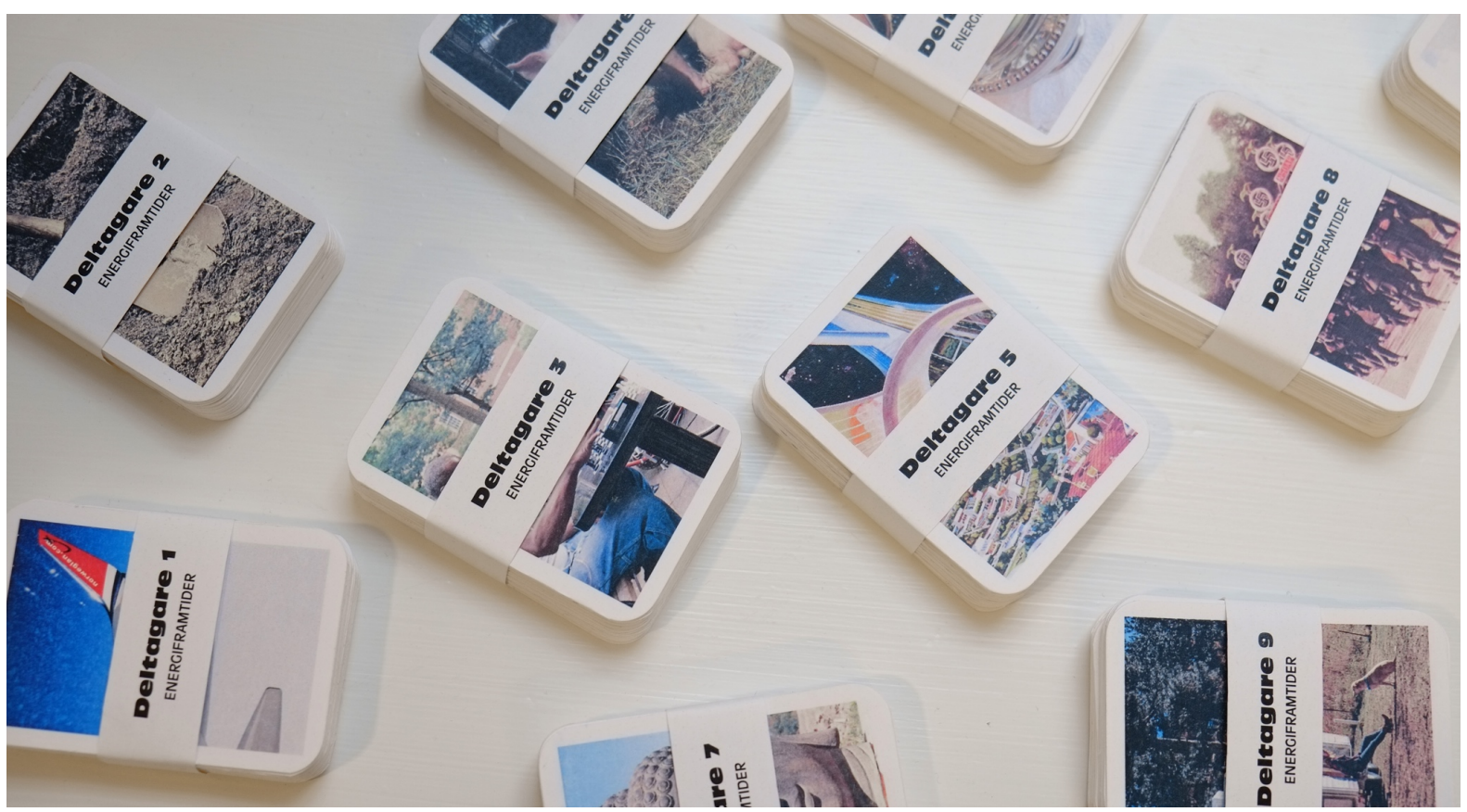

Figure 2 The cards used as the second trigger material.

\subsection{Trigger material 3}

The third trigger material comprised seven fictitious headline posters, representing possible configurations of Legato. The headlines were inspired by artistic explorations ${ }^{4}$ of futures and presents. The headlines were designed to spur reactions, to confront the participants with possible futures as if they were already here. The main reason for giving the futures-as-present the shape of headlines was that we wanted to use a familiar form but avoid images. Through this the participants did not have to spend time making sense of the form before making sense of the content (to the extent that these two can be separated). The avoidance of images aimed to activate the participant's own imagination in the sense-making process, thus decreasing the risk that participants distanced themselves from the content because they did not like or believe in our way of visually representing it. During the workshop, the participants were first invited to individually place post-its with their immediate thoughts at the different posters. This was followed by a discussion, after which the participants were divided into smaller groups to discuss more in depth one of the headline posters and its implications.

\subsection{Trigger material 4}

The fourth trigger material consisted of "fill-in-the-blanks" posters, where the participants themselves filled in the missing words. The material was created to support reflections, but also as a playful and co-creative ending to the workshop, inviting the participants to 'check out' while creating decrees about the present and future.

\footnotetext{
${ }^{4}$ Examples include the project "Wish you were here? Postcards from the Future" by Robert Graves and Didier Madoc-Jones, and Barbara Kruger's collages.
} 


\subsection{Workshop participants}

The workshop was arranged in May 2017, with 9 participants and conducted in Stockholm, Sweden. The participants were recruited by Kantar SIFO ${ }^{5}$ from a database of people willing to be part of discussion groups and who had indicated they were interested in environmental issues. Five were women, four were men and the participants were between 27 and 70 years old. Besides their mutual environmental interest, the participants were recruited to have a spread in interests and values. The two-and-a-half hour workshop was arranged during the evening and followed a workshop schedule that was open for the participants' discussions and reflections as interests shifted.

\section{Engaging with the trigger materials}

In this section, we describe some examples of the discussions that took place and provocations that the trigger materials created, with a focus on our perception of how the participants expressed their thoughts on lost and found.

\subsection{Trigger material 1}

In the beginning of the workshop, when the participants presented themselves and talked about their climate footprints, most of them were uncomfortable or even distressed, since (all but one of them) had larger footprints than they had anticipated:

"I was surprised, I thought I would be much lower, I've always seen myself like a hero, and then I'm just average. I thought everyone else were much more environmental villains compared to me." Participant 5

The participants' results in the climate footprint calculation carried out prior to the workshop ranged from 7.2 to $19.2 \mathrm{CO}_{2} \mathrm{e}$. All the participants had environmental concerns and tried to consume less or make more sustainable choices in their everyday lives. Furthermore, the climate footprint calculator results had surprised them, showing how large a portion of their footprints came from flying or housing, which they had previously not been aware of. There was a sensed tension when discussing their climate footprints and a subdued atmosphere around the table. When the goal of $0.6 \mathrm{CO}_{2} \mathrm{e}$ was presented there were exclamations of surprise and frustration.

\subsection{Trigger material 2}

When presented with the second trigger material (see Figure 3), the part focusing most clearly on lost and found, some of the participants moved between hope and despair as they navigated through different possibilities. Having previously expressed anger with their current footprints, some of the participants turned around and became positive as they realised that they would perhaps not miss so many of their current lifestyle choices. Instead, they expressed wishes for the non-material and the simple, as expressed in the following quote:

"An increase in non-material phenomena, experiences rather than buying things, things you do together, there was this picture with dancing people for example. Playing games, camping, things that do not require so much resources." Participant 2

Several participants also thought that we would live healthier in the future, as well as finding calmness and fulfilment in things closer to home. Some participants expressed hope in the development of new technologies including new types of foods. When it came to losses and fears, many expressed sadness over the loss of travelling, and fears for a more insecure and unstable future, with potentially more conflicts over resources, climate fugitives and irreversible waste from our current affluent society.

\footnotetext{
${ }^{5}$ Kantar SIFO is a company working with opinion and social research, surveys and recruitment for different polls.
} 
Some of the images, like those depicting for example military marching, were difficult for the participants to relate to and were interpreted differently. Some participants were confused with how the four fields of the matrix were to be interpreted as what they thought would happen or what they wanted to happen. Clearly, there was a tension between these two. Some participants had a need to also understand how the transition would happen, and even though they could envision alternative futures, they got stuck in not understanding how the necessary changes could possibly take place. Some participants expressed wishes regarding clear directions from governments and policy makers with new laws, regulations and even rationing of, for example, fossil fuels.

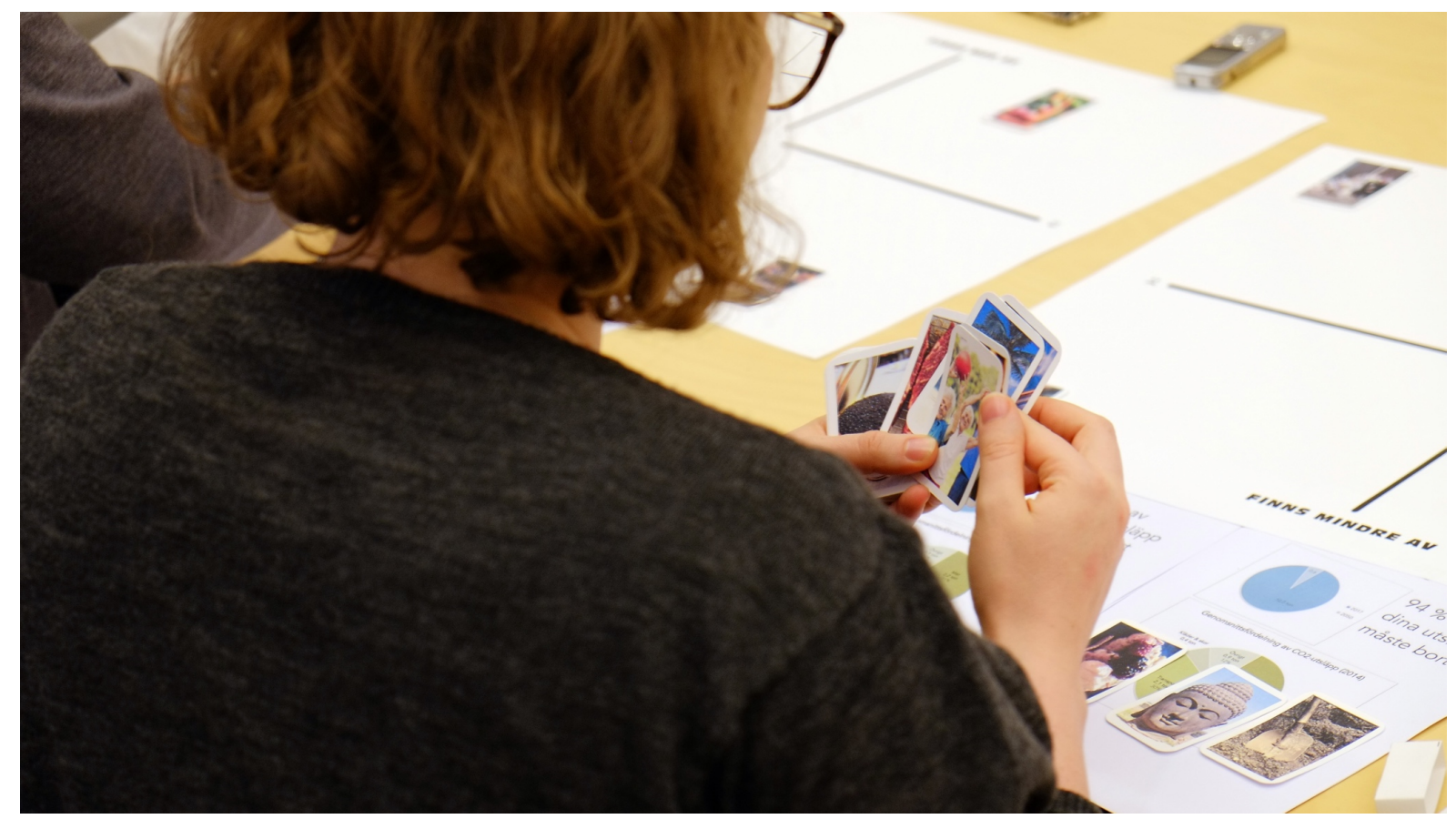

Figure 3 Participant reflecting and placing cards in the $2 \times 2$ matrix.

\subsection{Trigger material 3}

The third trigger material (see Figure 4) was the headline posters, and these also stirred up a fair amount of emotion, both negative and positive. When asked for first impressions, "Tonight the last airplane took off" was the headline poster that received the most attention. For some it was preposterous, a fable:

"It will never happen, [...] unless it is a world war. It is too black and white, there will always be exceptions. There will always be airplanes, even if it was decided that we ordinary people are not allowed to fly. There would be military or unquestionable transports of medicine." Participant 9

Many expressed sadness and loss of what the lack of travelling would lead to, but some also expressed positive feelings if flying were forbidden, potentially making train trips better and cheaper. One participant raised the question whether the reachable world would shrink whereby understandings of other cultures and customs might diminish. In the collective discussion around the headline posters, further topics were brought up, as if the posters had set in motion thinking about connected matters. One example was a discussion around self-sufficiency that prompted the participants to discuss working hours and a larger shift of time perception in society.

After a vote around the table, three of the headlines were chosen to be discussed in more depth in smaller groups ("Last airplane taking off tonight", "10 steps towards increased self-sufficiency", "Stockholm's major road will become a place for urban farming"). In the smaller groups, several tensions and problematic dilemmas were brought up. Regarding airplane transportation, the participants discussed the difference between necessary flying (for example medicines in emergency situations) and unnecessary flying (for example Thailand vacations). Other travel practices, such as 
train trips, were discussed as alternatives and the participants pointed out that appreciation of travel time could be an alternative value to promote. Also appreciating holiday time in your home town was pointed out as an alternative to unnecessary flying. Furthermore, the poster "Introducing meat tax" was discussed as a very realistic headline that actually could be implemented already this year. In order for a meat tax to have effect, the participants felt that the level of this tax would need to be very high. Furthermore, the participants also discussed the need for rationing fossil fuels and comparisons were made to how this took place in the 1970s and how well it worked at that time.

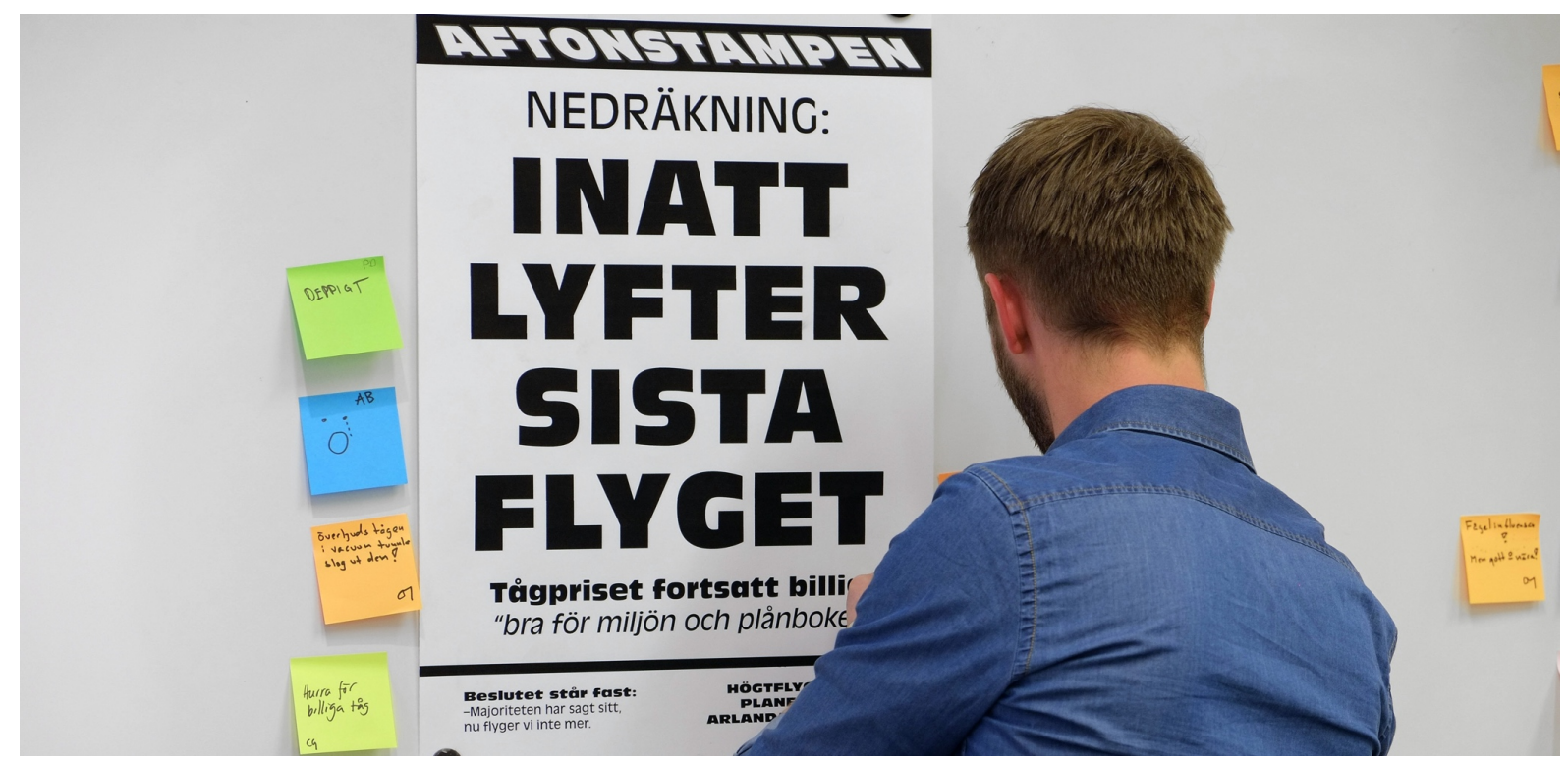

Figure 4 Participant writing down his first impressions of the headline poster stating: "Last airplane taking off tonight".

\subsection{Trigger material 4}

In the last exercise, the participants created their own posters of possible future headlines from newspapers and magazines (see Figure 5). Several of the participants put the message: "For the sake of my children and grandchildren, I refrain from flying, car-driving and eating meat".

Finally, there was an open discussion around the table where the participants were encouraged to talk about how they had experienced the workshop as a whole. Some described it as being intense and thought provoking, as expressed in the following quote:

"The uninhibited consumption our generation has experienced will never be relived. We are standing at a crossroad, we can't continue like this. We have to end it. I almost feel like an old dinosaur. Soon the comet will come and then everything will be changed." Participant 1

But many of the participants also expressed feelings of hope and positive outlooks for the future, even though some of them had come to the workshop with negative feelings about their own footprint and despair related to the seemingly impossible task of changing society into a more sustainable one. Some of the participants expressed gratefulness in being part of the workshop and claimed that they had learnt many new things and acquired interesting ideas. As a summary, the whole group, despite being diverse except for their engagement in environmental issues, seemed to gain a thirst for knowledge and a context to talk about climate change and the future. 


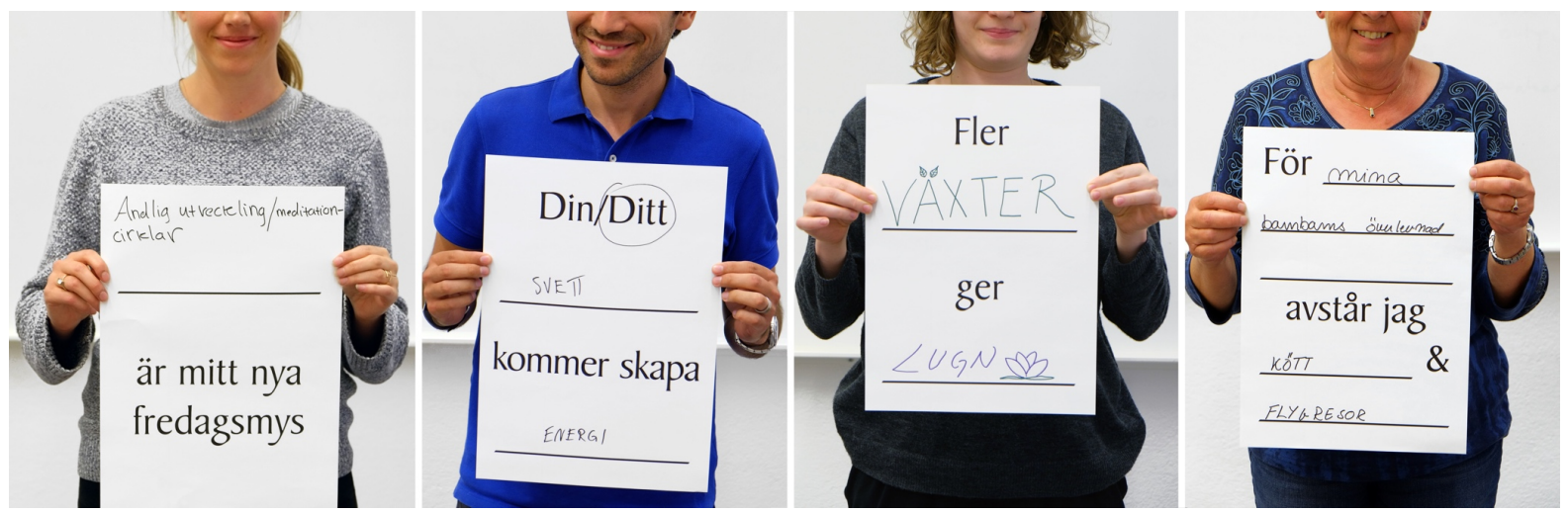

Figure 5 Participants with the posters they made, from left to right: "Spiritual development/meditation circles is my new Friday family time, "Your sweat will create energy", "More plants give peace of mind" and "For the survival of my grandchildren I refrain from meat and flying".

\section{Discussion: Balancing act}

The trigger materials, i.e. research devices, used in this research project were designed to engage users in exploring and articulating lost and found, deliberately developed to be both provocative and affirmative. To start with, we used forerunners of practices identified as important for sustainability transitions. Meeting these forerunners in their homes to discuss their everyday lives enabled us to access their knowledge about how to solve everyday issues and to use this as design inspiration for the trigger materials. In these in-home-interviews, we focused on understanding the practices, including how they emerged and were maintained, i.e. how links were broken and established. As practices are dynamic and unstable, as well as bundled together, it is crucial to understand their interconnections (Shove and Walker, 2010). Even though practices can never be controlled, they can be orchestrated (ibid.) and since our intention in this project was to understand how to push sustainability transitions, we were specifically interested in understanding how the forerunners had made new practice bundles and how these changes came about.

From the interviews and analysis of Four Futures we could identify a large number of entry points to discuss energy futures with our workshop participants. As is often the case in a design process, the real challenge was not in finding material but in deciding how to conceptualise this into working categories. In this project, we deliberately designed to balance and bridge (see Sanders \& Stappers, 2012) the affirmative, the mundane everyday here and now, with the provocative, imagining fundamentally different futures. However, it is as challenging to shift from understanding the present to construct possible futures as it is to think outside the current norms and values to develop future ideas.

The trigger material developed for the workshop included questioning norms as well as discussing established and well-known everyday practices. This balance between provoking current everyday life while still being affirmative to how it is actually conducted, is what we tested in the design of the trigger material, with the aim of engaging the participants in exploring lost and found in a sustainable future. For example, in trigger material 1, each workshop participant assessed their current $\mathrm{CO}_{2} \mathrm{e}$ emissions. This sensitizing device worked well to create a space for reflection, even prior to the workshop, and most of the participants were provoked by their individual results. To use an audit can create a space for reflection that might contribute to more sustainable practices (Hargreaves, 2011). When faced with the need to decrease $\mathrm{CO}_{2}$ e emissions, from their individual results to the goal of 0.6 , some of the participants felt frustrated as they did not know what they could possibly do to reach such a low level. However, as the workshop continued, it was clear that the individual assessment had caused reflection of possible futures, with new things found even whilst keeping links to current everyday lives.

With the second trigger material, it was clear that the co-design approach (which enabled the participants to first individually reflect and then tell the group about their card selections and 
placements) worked well as a bridge between participants' present practices (some which might be lost) and envisioned future possibilities (which in many cases were new found values). The images also worked to facilitate the transformation from concrete details to bigger and more abstract pictures. However, some of the images, for example those that caused reflection on war and totalitarian societies, were simply discarded by some as they were considered too provocative. When too provocative, the participants could not (or did not want to) connect to the material and no reflections were initiated. In this case, when the images were too provocative, the trigger material did not work so well, which is in line with research on climate communication (Kollmuss \& Agyeman, 2002). Some participants got stuck in trying to figure out whether the matrix should depict what would happen, or what they wanted to happen in the future. Even though this ambiguity could be hampering, we believe that it is needed, since it can capture both fears and aspirations.

The third trigger material, the posters with deliberately strong headlines, spanned both the provocative and the affirmative. Some of the headlines pushed the participants quickly into future possibilities and they had no problems envisioning drastic changes of current regulations, laws and taxes to enable reaching targets, since this would ensure that they as individuals would not be the only ones breaking the norm. As many of the required changes are actually uncomfortable and inconvenient, provocations can be needed for this push. It was clear in the workshop that citizens desire that policy makers take actions and guide. The headlines also gave rise to many discussions and to some revelations of new things that might be found in a sustainable future - later displayed when the participants made their own headlines (trigger material 4). Here some of their concerned losses related to what they would abstain from in order to save the world for their children or grandchildren. However, many focused on newly found things, such as more time, more spirituality and new solutions. For some of the participants it was also difficult to understand how some suggested practices could possibly be implemented. The struggle to connect visions of desirable futures with change of existing everyday practices, and the need to understand the complete and complex implementation, is not uncommon for those who are not used to creative thinking and creative processes. We can also see that the trigger materials one by one might not help in bridging the tangible-present-local to the abstract-future-global, but in unison they helped the participants, in different ways, to make movements in time, place and possibilities.

\section{Conclusions}

In this project we have, through practice-based design research and with a transition design posture, designed tools that we believe can be useful to initiate dialogues and reflections on the future. We can see that the trigger materials worked well as research devices, and that they managed to, if not bridge, at least allow for a co-existence of provocative and affirmative approaches.

This research project has had its focus on Sweden and we have carried out just one workshop in Stockholm - we have had no ambition of painting a complete picture of all possible images of futures people might have. Moreover, we have developed only one set of trigger materials. We see great potential for developing different trigger materials that could be tested in different types of workshops, and to change the type of people participating. It could be interesting to conduct workshops with those in power positions, like politicians and authority leaders. We believe that a further development of the trigger material presented in this paper could be useful as workshop material in, for example, non-profit organisations or study circles, where there could be interest for creative explorations of lost and found.

It is clear that there is a discrepancy between the actions needed to reach the target for a sustainable energy system and the images people have of their existing and future energy use. Even so, people in Sweden are willing to engage in issues around transitions but many do not know what to do or where to start. We believe it is important to widen the horizon to help people understand that an energy system is not set in stone, and that many different futures are possible. 


\section{Acknowledgements}

This project has been funded by the Swedish Energy Agency (P40238-1). We want to thank all the cocreators in the project that we met in interviews and in the provocation workshop. A special thanks Andrejs Ljunggren and Gabriel Kanulf.

\section{References}

Bell, G., Blythe, M., \& Sengers, P. (2005). Making by making strange: Defamiliarization and the design of domestic technologies. Title. ACM Transactions on Computer-Human Interaction (TOCHI), 12(2), 149173.

Energimyndigheten. (2016). Fyra Framtider - Energisystemet efter 2020. ET2016:04.

Ereaut, G., \& Segnit, N. (2006). Warm words: How we are telling the climate story and can we tell it better?

Forty, A. (1986). Objects of desire: Design and Society Since 1750. London: Thames and Hudson Ltd.

Geels, F. W. (2010). Ontologies, socio-technical transitions (to sustainability), and the multi-level perspective. Research Policy, 39(4), 495-510. https://doi.org/10.1016/j.respol.2010.01.022

Giddens, A. (2011). The Politics of Climate Change. Cambridge: Polity Press.

Hargreaves, T. (2011). Practice-ing behaviour change: Applying social practice theory to pro-environmental behaviour change. Journal of Consumer Culture, 11(1), 79-99.

https://doi.org/10.1177/1469540510390500

Harper, D. (2002). Talking about pictures: A case for photo elicitation. Visual Studies, 17(1), 13-26. https://doi.org/10.1080/14725860220137345

IPCC 2014. (2014). Summary for Policymakers. Climate Change 2014: Synthesis Report. Contribution of Working Groups I, II and III to the Fifth Assessment Report of the Intergovernmental Panel on Climate Change. https://doi.org/10.1017/СВ09781107415324

Irwin, T., Kossoff, G., \& Tonkinwise, C. (2015). Transition Design Provocation. Design Philosophy Papers, 13(1), 3-11. https://doi.org/10.1080/14487136.2015.1085688

Kemp, R., Loorbach, D. A., \& Rotmans, J. (2007). Transition management as a model for managing processes of co-evolution towards sustainable development. International Journal of Sustainable Development and World Ecology, 14(1), 78-91.

Kollmuss, A., \& Agyeman, J. (2002). Mind the Gap: Why do people act environmentally and what are the barriers to pro-environmental behavior? Environmental Education Research, 8(3), 239-260. https://doi.org/10.1080/13504620220145401

Lowe, T. D. (2006). Is This Climate Porn? How Does Climate Change Communication Affect Our Perceptions and Behavior? Tyndall Centre for Climate Change Research Working Paper, 98(December), 43.

Lury, C., \& Wakeford, N. (2012). Inventive Methods. London and New York: Routledge. https://doi.org/10.4324/9780203854921

Miller, T. R., Baird, T. D., Littlefield, C. M., Kofinas, G., Chapin III, F. S., \& Redman, C. L. (2008). Epistemological Pluralism: Reorganizing Interdisciplinary Research. Ecology and Society, 13(2).

Nelson, H. G., \& Stolterman, E. (2003). The Design Way: Intentional change in an unpredictable world: Foundations and fundamentals of design competence. London: The MIT Press.

Norgaard, K. M. (2011). Living in denial: Climate Change, Emotions, and Everyday Life. London: MIT Press.

Randall, R. (2009). Loss and Climate Change: The Cost of Parallel Narratives. Ecopsychology, 1(3), 118-129. https://doi.org/10.1089/eco.2009.0034

Reckwitz, A. (2002). Toward a theory of social practices. European Journal of Sociology, 5(2), 243-263.

Robinson, J. (2004). Squaring the circle? Some thoughts on the idea of sustainable development. Ecological Economics, 48(4), 369-384. https://doi.org/10.1016/j.ecolecon.2003.10.017

Robinson, J. (2008). Being undisciplined: Transgressions and intersections in academia and beyond. Futures, 
4O(1), 70-86. https://doi.org/10.1016/j.futures.2007.06.007

Sanders, L. (2008). An evolving map of design practice and design research. Interactions, 15(1), 13-17. https://doi.org/10.1145/1409040.1409043

Sanders, L., \& Stappers, P. J. (2012). Convivial design toolbox: generative research for the front end of design. Amsterdam: BIS Publishers.

Sanders, L., \& Stappers, P. J. (2014). Probes, toolkits and prototypes: Three approaches to making in codesigning. CoDesign, 10(1), 5-14. https://doi.org/10.1080/15710882.2014.888183

Schneider-Mayerson, M. (2017). "Climate change fiction", in American Literature in Transition: 2000 - 2010. (R. Greenwald Smith, Ed.). Cambridge University Press.

Schön, D. (1983). The Reflective Practitioner. Pediatrics, 116(6), 1546-52. https://doi.org/10.1542/peds.20050209

Shove, E., \& Walker, G. (2010). Governing transitions in the sustainability of everyday life. Research Policy, 39(4), 471-476. https://doi.org/10.1016/j.respol.2010.01.019

Steffen, W., Richardson, K., Rockström, J., Cornell, S., Fetzer, I., Bennett, E., ... Carpenter, S. (2015). Planetary boundaries: Guiding human development on a changing planet. Science (New York, N.Y.), 348(6240), 1217. https://doi.org/10.1126/science.aaa9629

Stirling, A. (2011). Pluralising progress: From integrative transitions to transformative diversity. Environmental Innovation and Societal Transitions, 1(1), 82-88. https://doi.org/10.1016/j.eist.2011.03.005

Stocker, T., Qin, D., Plattner, G., Tignor, M., Allen, S., Boschung, J., Nauels, A., Xia, Y., Bex, V., and Midgley, P. . (2013). Climate Change 2013: The Physical Science Basis. Working Group I Contribution to the Fifth Assessment Report of the Intergovernmental Panel on Climate Change. Summary for Policymakers.

Tonkinwise, C. (2016). Failing to Sense the Future: From Design to the Proactionary Test Drive. Social Research, 83(3), 597-624.

UNEP. (2017). The Emissions Gap Report 2017: A UN Environment Synthesis Report. Nairobi. https://doi.org/ISBN 978-92-9253-062-4

Warde, A. (2005). Consumption and Theories of Practice. Journal of Consumer Culture, 5(2), 131-153. https://doi.org/10.1177/1469540505053090

Weber, E. U. (2010). What shapes perceptions of climate change? WIREs Clim Change, 1, 332-342. https://doi.org/10.1002/wcc.41

About the Authors:

Mia Hesselgren is a PhD Candidate in Design and Sustainability. Her research focuses on how methods in service design, strategic design and collaborative design can be used for sustainability transitions.

Elina Eriksson is an assistant professor in Human-Computer Interaction with a specialization in sustainability. She is interested in the social construction of denial and wants more people to engage in sustainability issues - even if it hurts.

Josefin Wangel is an un-disciplinary researcher and educator at SLU Swedish University of Agricultural Sciences with a passion for socio-material systems thinking, critical theory and speculative approaches, particularly to explore how, when and if sustainable + urban can be combined.

Loove Broms is a researcher and associate professor in interaction design. He is interested in discursive artefacts, narratives and meaning-making in relation to the research topic of sustainability and design. 